\title{
BMJ Open The challenge of vaccinating adults: attitudes and beliefs of the Canadian public and healthcare providers
}

\author{
D M MacDougall, ${ }^{1,2}$ B A Halperin, ${ }^{1,3,4}$ D MacKinnon-Cameron, ${ }^{1} \mathrm{Li} \mathrm{Li},{ }^{1}$ \\ S A McNeil, ${ }^{1,5}$ J M Langley, ${ }^{1,4,6}$ S A Halperin ${ }^{1,4,7}$
}

To cite: MacDougall DM, Halperin BA, MacKinnonCameron D, et al. The challenge of vaccinating adults: attitudes and beliefs of the Canadian public and healthcare providers. BMJ Open 2015;5:e009062. doi:10.1136/bmjopen-2015009062

- Prepublication history for this paper is available online. To view these files please visit the journal online (http://dx.doi.org/10.1136/ bmjopen-2015-009062).

Received 11 June 2015 Revised 25 August 2015 Accepted 3 September 2015

CrossMark

For numbered affiliations see end of article.

Correspondence to Dr SA Halperin; scott. halperin@dal.ca

\section{ABSTRACT}

Objectives: Vaccine coverage for recommended vaccines is low among adults. The objective of this study was to assess the knowledge, attitudes, beliefs and behaviours of adults and healthcare providers related to four vaccine-preventable diseases and vaccines (diphtheria-tetanus-pertussis, zoster, pneumococcus and influenza).

Design: We undertook a survey and focus groups of Canadian adults and healthcare providers (doctors, nurses, pharmacists). A total of 4023 adults completed the survey and 62 participated in the focus groups; 1167 providers completed the survey and 45 participated in the focus groups.

Results: Only $46.3 \%$ of adults thought they were up-to-date on their vaccines; $30 \%$ did not know. In contrast, $75.6 \%$ of providers reported being up-todate. Only $57.5 \%$ of adults thought it was important to receive all recommended vaccines (compared to $87.1-91.5 \%$ of providers). Positive attitudes towards vaccines paralleled concern about the burden of illness and confidence in the vaccines, with providers being more aware of disease burden and confident in vaccine effectiveness than the public. Between $55.0 \%$ and $59.7 \%$ of adults reported willingness to be vaccinated if recommended by their healthcare provider. However, such recommendations were variable; while $77.4 \%$ of the public reported being offered and $52.8 \%$ reported being recommended the influenza vaccine by their provider, only $10.8 \%$ were offered and $5.6 \%$ recommended pertussis vaccine. Barriers and facilitators to improved vaccine coverage in adults, such as trust-mistrust of health authorities, pharmaceutical companies and national recommendations, autonomy versus the public good and logistical issues (such as insufficient time and lack of vaccination status tracking), were identified by both the public and providers.

Conclusions: Despite guidelines for adult vaccination, there are substantial gaps in knowledge and attitudes and beliefs among both the public and healthcare providers that lead to low vaccine coverage. A systematic approach that involves education, elimination of barriers and establishing and improving infrastructure for adult immunisation is required.

\section{Strengths and limitations of this study}

- Mixed methodology including survey and focus groups.

- Large national representative survey.

- Healthcare providers and the general public.

- Whether Canadian data are generalisable to other countries with different vaccine programmes.

- Self-reporting vaccine coverage status.

\section{INTRODUCTION}

While universal immunisation of children is now part of routine global healthcare and has led to substantial reductions in vaccinepreventable diseases, immunisation of adults and control of infectious disease morbidity and mortality substantially lags behind..$^{1-4}$ Several vaccines routinely given during childhood are recommended as boosters for adults such as tetanus-diphtheria-acellular pertussis vaccine, and other vaccines are specifically targeted to adults (eg, zoster vaccine, influenza vaccine. ${ }^{5-7}$ Multiple factors have been identified that influence immunisation uptake among adults including social influences, disease-related and vaccine-related factors, general attitudes toward health and vaccines, habit, awareness and knowledge, practical barriers and motivators, and altruism. $^{8}$ Barriers to improved immunisation coverage in adults include misperceptions among the public and healthcare providers (HCPs) that vaccines are just for children and logistical issues related to vaccine delivery, including lack of vaccine-specific HCP visits, inability to determine immunisation status and lack of funding for adult vaccines and vaccine visits. ${ }^{9}$ Although strategies have been identified to meet the challenge of low vaccine coverage in adults, ${ }^{1}$ little progress has been achieved.

In Canada, vaccine recommendations are made by the National Advisory Committee 
on Immunization (NACI); vaccine programmes, however, are implemented independently by each provincial/territorial government. Some vaccines, such as influenza vaccine, are provided free of charge in all provinces/territories; others, such as pertussis and pneumococcal vaccine are provided by some but not all jurisdictions and zoster vaccine is not provided by any jurisdiction. As the concept of an adult immunisation platform becomes increasingly part of public health planning, ${ }^{1}{ }^{10}$ we sought to develop a better understanding of the knowledge, attitudes, beliefs and behaviours of HCPs providing care to adults in Canada and the general public. Specifically we explored general issues regarding adult immunisation and specific information related to four diseases and the associated vaccines recommended for routine use in adults (influenza, pertussis, pneumococcus, zoster). A geographically representative national survey and focus groups involving the general public and HCPs who vaccinate was undertaken.

\section{METHODS}

We used a mixed method, sequential, explanatory design consisting of quantitative data collection and analysis (survey) followed by qualitative data collection and analysis (focus groups); ${ }^{11} 12$ details of the methodology have previously been published. ${ }^{13}$ Focus groups were chosen rather than interviews in order to get a concentrated set of observations over a short period of time. As well, interaction among participants increases their sense of cohesiveness and increases their willingness to explore and clarify individual and shared perspectives. ${ }^{14}$

\section{Quantitative stage (survey)}

The survey was developed using the Awareness Adherence Model. ${ }^{15}$ The content validity of individual questions as well as the content validity of the entire questionnaire were evaluated by a panel of experts comprised of infectious diseases physicians. Each item was rated using a standard content validity index with a fourpoint ordinal rating scale, where 1 indicated irrelevance and 4 high relevance. Items that received a score of 3 or 4 were judged to have content validity. Items that did not achieve the required minimum agreement of experts were eliminated or revised. Test-retest reliability was assessed by having five healthcare providers complete the questionnaires at two different points in time. A correlation coefficient was calculated to compare the two sets of responses; a coefficient $>0.70$ was interpreted that the questionnaire responses were consistent. The survey was then piloted on a convenience sample of 299 of the 1250 attendees at the 2010 Canadian Immunization Conference held in Quebec City. The national public survey reported here was administered by Leger Marketing (Montreal, Quebec, Canada), which maintains email addresses for 350000 Canadian adults who are representative of the Canadian general population for the purpose of participating in market and other research. Sampling was based on regional representation across the country, age, gender and urban and rural residence. A subset of HCPs within this database was invited to participate in the HCP survey. Sampling was based on regional representation, age, gender, urban and rural practice, and specialty (general practice physicians, internal medicine specialists, nurses and pharmacists). Inclusion criteria were being in practice for a minimum of 3 years and responsibility for immunisation delivery to adults and/or patient consultation concerning vaccines in their province or territory. Participants received an email invitation to the survey outlining the purpose of the study, its voluntary nature and the time commitment involved. Consent to participate was implied by completion of the web-based survey.

For the public survey, a sample size of 4000 adults was calculated to provide an acceptable precision by region (95\% CI around the point estimate) of $\pm 5 \%$. For the HCP survey, a sample size of 500 family physicians and 400 pharmacists was calculated to provide a precision (95\% CI around the point estimate) of $\pm 5 \%$; a sample size of 100 internal medicine specialists and 200 nurses was calculated to provide a precision of $\pm 5-10 \%$ for each practitioner type. The first level of analysis comprised a review of the descriptive, summative statistics for trends in the data. The second level of analysis involved tests of association. Data were divided by public and by HCP profession (physician, nurse and pharmacist) and locale (province/territory). Continuous variables were presented by summary statistics (ie, mean and SE) and the categorical variables by frequency distributions (ie, frequency counts, percentages and their two-sided 95\% exact binomial CIs). Differences in survey responses between groups were assessed using Fisher's exact tests. For continuous variables, logistic regression was used. Associations between attitude questions, behavioural responses and demographics were estimated using ordinal logistic regression or Fisher's exact tests. p Values $<0.05$ were considered statistically significant.

\section{Qualitative stage (focus groups)}

Focus groups were administered by Leger Marketing using trained, experienced facilitators in multiple locations across Canada using a semistructured facilitation guide developed by the academic investigators (DMM, $\mathrm{BAH})$, who attended selected sessions. Researchers (DMM, BAH) had experience and/or training in qualitative methods. All members of the research team were vaccinologists with public health experience and a vested interest in promoting immunisation within the public domain. None of the investigators had prior relationships with any of the participants in the study. For the public, six face-to-face focus groups and two 'virtual' web-based focus groups were undertaken. For HCPs, six face-to-face focus groups, two 'virtual' web-based focus groups and four one-on-one interviews were undertaken. Regional representation was sought with a balance of rural and urban residence for the public and large and small urban 
areas, suburban and rural practices for HCPs. Inclusion criteria for the public survey were being an adult aged 21-65 years, with two-thirds of participants per focus group having frequent contact with children. Inclusion criteria for HCP participation were routinely providing immunisations or advice about immunisation to patients and being in practice for a minimum of 3 years. HCPs included nurses, pharmacists and physicians (including general practitioners, internists and emergency room physicians).

Discussion guides developed by the academic investigators for both HCPs and the general public included questions that probed for information regarding general perceptions and experiences with adult vaccination and reasons for receiving or not receiving adult vaccines. The guides were developed using the results of the national survey of the Canadian public and HCPs and pilot-tested prior to implementation. The questioning route used for the focus groups followed the guidelines as outlined in Krueger and Casey. ${ }^{16}$ Topics that were explored were knowledge of adult vaccination, vaccine recommendations, attitudes about side effects and delivery of adult vaccines. All focus groups were recorded and transcribed verbatim. A debriefing with the moderator team took place immediately following the focus group. Thematic analysis was initiated concurrent with the first focus group as previously described. ${ }^{13}$ Two research team members (DMM, KW) individually coded the data using the process of thematic analysis. Both read all transcripts to generate an initial set of codes and then collated according to similarities and related patterns as well as for differences, followed by combining and cataloguing similar patterns into subthemes (NUD*IST software version N9, Sage Publications Ltd, London, UK). The themes were refined through ongoing analysis. Team members met regularly to review the emerging themes and to achieve consensus. Focus groups continued until saturation was achieved. Following analysis, the findings were reviewed in relationship with the existing literature.

\section{RESULTS \\ Survey}

A total of 4023 adults completed the public survey; $2252(56 \%)$ were men and $1771(44 \%)$ were women (table 1). More respondents were 45-54 years of age and fewer were younger than 25 years and 75 years and older. For the HCP survey, there were 1167 respondents, comprising $42.8 \%$ family physicians, $5.6 \%$ internists, $34.3 \%$ pharmacists and $17.3 \%$ nurses. Most (83.9\%) practiced in an urban/suburban setting. Ninety-three per cent of physicians, $41 \%$ of pharmacists and $54 \%$ of nurses provided direct patient care at least $75 \%$ of the time.

Only $46.3 \%$ of Canadian adults reported thinking that they were up to date on all of their adult vaccinations; $30.0 \%$ did not know (table 1). More HCPs reported being up to date: $83.7 \%$ of nurses, $69.2-78.0 \%$ of physicians and $69.5 \%$ of pharmacists. Up-to-date immunisation status reflected attitudes toward adult immunisation; $87.1-91.5 \%$ of HCPs agreed or strongly agreed that it was important to receive all recommended adult vaccinations (table 2) compared to only $57.5 \%$ of the general public (table 3). The proportion of the public and HCPs who agreed with the statement that vaccines are more important for children than adults was similar (40.6-46.9\% of HCPs and $48.3 \%$ of the public). Similar proportions agreed that recommended adult vaccines should be publicly funded (81.8-91.6\% of HCPs and $78.1 \%$ of the public). HCPs were more concerned than the general public about the burden of illnesses prevented by the vaccines and the effectiveness of the vaccines available to prevent them. Over $92 \%$ of HCPs believed that influenza, pneumococcal pneumonia and shingles could have an important impact on the health of adults; $82.8-86.7 \%$ believed that about pertussis. In contrast, $84.2 \%$ and $88.8 \%$ of the public believed that influenza and pneumococcal pneumonia, respectively, had an important impact and only $74.0 \%$ and $56.3 \%$ thought that about shingles and pertussis, respectively. Confidence in the effectiveness of the vaccines for those infections was correspondingly higher among HCPs than the public (tables 2 and 3, respectively).

Only 25\% of Canadian adults reported that their HCP had informed them of which adult vaccines they should receive; while $77.4 \%$ reported being offered and $52.8 \%$ reported being recommended influenza vaccine, only $10.8 \%$ were offered and $5.6 \%$ recommended pertussis vaccine. Approximately half of the public respondents reported being immunised with influenza vaccine and $14.1 \%$ reported being immunised with pertussis vaccine. Importantly, $55.0-59.7 \%$ reported willingness to be immunised with the four adult vaccines if their HCP recommended it (table 3 ).

With regard to logistical issues, over $91 \%$ of HCPs believed it was important to inform adult patients about the benefits and risks of adult vaccinations; however, just over half believed it was difficult to keep current with vaccine recommendations (table 2). Most HCPs reported having sufficient time to provide vaccination and having adequate storage facilities; however, adequacy of reimbursement was an issue for physicians and pharmacists (but not nurses). Most HCPs believed it was important to use scheduled or unscheduled patient encounters for other health reasons to ask about vaccine status; a minority of HCPs used visits of children as an opportunity to update vaccinations on accompanying adults. Approximately one-third of physicians and nurses and only $11.3 \%$ of pharmacists had a system in their practice to identify adults who had not received recommended vaccines. Over $75 \%$ of HCPs agreed that there should be a national electronic registry to track all vaccines administered to an individual. This type of registry was supported by the public; $75.8 \%$ of the respondents agreed 
Table 1 Characteristics of respondents to the national survey of Canadian healthcare providers and the public

\begin{tabular}{|c|c|c|c|c|c|}
\hline \multirow[b]{2}{*}{ Characteristic } & \multirow[b]{2}{*}{$\begin{array}{l}\text { Nurses } \\
(\mathrm{n}(\%))\end{array}$} & \multicolumn{2}{|c|}{ Physicians (n (\%)) } & \multirow[b]{2}{*}{$\begin{array}{l}\text { Pharmacists } \\
\text { (n (\%)) }\end{array}$} & \multirow{2}{*}{$\begin{array}{l}\text { Canadian } \\
\text { Public } \\
(\mathrm{n}(\%))\end{array}$} \\
\hline & & $\begin{array}{l}\text { Family } \\
\text { Physicians }\end{array}$ & Internists & & \\
\hline Profession & 202 (17.3) & $500(42.8)$ & $65(5.6)$ & 400 (34.3) & Not applicable \\
\hline \multicolumn{6}{|l|}{ Sex } \\
\hline Female & $186(92.1)$ & $170(34.0)$ & $16(24.6)$ & 199 (49.8) & $1771(44.0)$ \\
\hline Male & $16(7.9)$ & $330(66.0)$ & 49 (75.4) & 201 (50.3) & $2252(56.0)$ \\
\hline \multicolumn{6}{|l|}{ Age } \\
\hline$\leq 24$ & $3(1.5)$ & - & - & $1(0.3)$ & 354 (8.8) \\
\hline$\overline{25}-34$ & $53(26.2)$ & $45(9.0)$ & $2(3.1)$ & $104(26.0)$ & $509(12.7)$ \\
\hline $35-44$ & $54(26.7)$ & $118(23.6)$ & $24(36.9)$ & $164(41.0)$ & $676(16.8)$ \\
\hline $45-54$ & 57 (28.2) & $210(42.0)$ & $25(38.5)$ & $99(24.8)$ & 933 (23.2) \\
\hline $55-64$ & 35 (17.3) & $127(25.4)$ & $14(21.5)$ & $32(8.0)$ & 790 (19.6) \\
\hline $65-74$ & - & - & - & - & $623(15.5)$ \\
\hline$\geq 75$ & - & - & - & - & $138(3.4)$ \\
\hline \multicolumn{6}{|l|}{ Province } \\
\hline British Columbia & $41(20.3)$ & $81(16.2)$ & $5(7.7)$ & $53(13.1)$ & $504(12.5)$ \\
\hline Alberta & 35 (17.3) & $56(11.2)$ & $7(10.8)$ & 45 (11.3) & 403 (10.0) \\
\hline Saskatchewan & $12(5.9)$ & $18(3.6)$ & $1(1.5)$ & $19(4.8)$ & $251(6.2)$ \\
\hline Manitoba & $12(5.9)$ & $18(3.6)$ & $5(7.7)$ & 17 (4.3) & $253(6.3)$ \\
\hline Ontario & $60(29.7)$ & $168(33.6)$ & $25(38.5)$ & 125 (31.3) & $1206(30.0)$ \\
\hline Quebec & $16(7.9)$ & $123(24.6)$ & $17(26.2)$ & $82(20.5)$ & $804(20.0)$ \\
\hline New Brunswick & $10(5.0)$ & $8(1.6)$ & $1(1.5)$ & $23(5.8)$ & $227(5.6)$ \\
\hline Nova Scotia & $10(5.0)$ & $18(3.6)$ & $3(4.6)$ & $22(5.5)$ & $220(5.5)$ \\
\hline Prince Edward Island & $1(0.5)$ & $2(0.4)$ & - & $2(0.5)$ & $46(1.1)$ \\
\hline Newfoundland & $5(2.5)$ & $8(1.6)$ & $1(1.5)$ & $12(3.0)$ & $109(2.7)$ \\
\hline \multicolumn{6}{|c|}{ Nature of practice (HCP) or residence (public) } \\
\hline Urban & $116(57.4)$ & $285(57.0)$ & $38(58.5)$ & $219(54.8)$ & $1691(42.0)$ \\
\hline Suburban & $46(22.8)$ & $132(26.4)$ & $18(27.7)$ & 125 (31.3) & $1471(36.6)$ \\
\hline Rural & $40(19.8)$ & $83(16.6)$ & $9(13.8)$ & $53(13.3)$ & $830(20.6)$ \\
\hline Do not know & - & - & - & - & $31(0.8)$ \\
\hline Involved in direct patient care $>75 \%$ & $109(54.0)$ & 469 (93.8) & $56(86.2)$ & $164(41.0)$ & Not applicable \\
\hline $\begin{array}{l}\text { Number of years providing vaccines; } \\
\text { mean (SD) }\end{array}$ & $9.7(7.3)$ & $19.5(8.9)$ & $15.9(4.5)$ & $6.1(7.5)$ & Not applicable \\
\hline \multicolumn{5}{|l|}{ Vaccines administered to adults/month } & Not applicable \\
\hline None & $13(7.1)$ & $5(1.0)$ & $3(4.7)$ & $14(4.2)$ & \\
\hline $1-5$ & $61(33.5)$ & $46(9.3)$ & $19(29.7)$ & $106(32.1)$ & \\
\hline $6-10$ & $38(20.9)$ & $101(20.4)$ & $17(26.6)$ & $95(28.8)$ & \\
\hline $11-20$ & $32(17.6)$ & 165 (33.3) & $14(21.9)$ & $82(24.8)$ & \\
\hline $21-50$ & $26(14.3)$ & $131(26.5)$ & $6(9.4)$ & $25(7.6)$ & \\
\hline$>50$ & $12(6.6)$ & $47(9.5)$ & $5(7.8)$ & $8(2.4)$ & \\
\hline Highest level of education & Not obtained & Not obtained & Not obtained & Not obtained & \\
\hline Elementary & & & & & $18(0.4)$ \\
\hline High school & & & & & $964(24.0)$ \\
\hline College & & & & & $1221(30.4)$ \\
\hline University diploma & & & & & $314(7.8)$ \\
\hline University baccalaureate & & & & & $1038(25.8)$ \\
\hline University masters & & & & & $355(8.8)$ \\
\hline University doctorate & & & & & $83(2.1)$ \\
\hline Prefer not to answer & & & & & $30(0.7)$ \\
\hline \multicolumn{6}{|c|}{ Do you think you are up to date on all your adult vaccinations? (\%) } \\
\hline Yes & 83.7 & 78.0 & 69.2 & 69.5 & $1861(46.3)$ \\
\hline No & 11.9 & 16.6 & 20.0 & 18.8 & $954(23.7)$ \\
\hline Do not know & 4.5 & 5.4 & 10.8 & 11.8 & $1208(30.0)$ \\
\hline
\end{tabular}


Table 2 Healthcare provider attitudes about adult immunisation

\begin{tabular}{|c|c|c|c|c|c|}
\hline Synopsis of statements & Agreement & $\begin{array}{l}\text { Nurses } \\
(\%)\end{array}$ & $\begin{array}{l}\text { Physicians } \\
(\%)\end{array}$ & $\begin{array}{l}\text { Pharmacists } \\
(\%)\end{array}$ & p Value \\
\hline \multirow{3}{*}{$\begin{array}{l}\text { It is important for adults to receive all } \\
\text { recommended vaccines according to } \\
\text { provincial guidelines }\end{array}$} & Strongly agree/agree & 87.1 & 90.6 & 91.5 & \multirow[t]{3}{*}{0.0085} \\
\hline & $\begin{array}{l}\text { Neither agree nor } \\
\text { disagree }\end{array}$ & 9.4 & 9.0 & 6.5 & \\
\hline & $\begin{array}{l}\text { Disagree/strongly } \\
\text { disagree }\end{array}$ & 3.5 & 0.4 & 2.0 & \\
\hline \multirow{3}{*}{$\begin{array}{l}\text { Vaccines are more important for children than } \\
\text { adults }\end{array}$} & Strongly agree/agree & 40.6 & 46.9 & 41.3 & \multirow[t]{3}{*}{0.1370} \\
\hline & $\begin{array}{l}\text { Neither agree nor } \\
\text { disagree }\end{array}$ & 16.8 & 19.5 & 21.0 & \\
\hline & $\begin{array}{l}\text { Disagree/strongly } \\
\text { disagree }\end{array}$ & 42.6 & 33.6 & 37.8 & \\
\hline \multirow{3}{*}{$\begin{array}{l}\text { Vaccines that are recommended for adults } \\
\text { should be available free of charge }\end{array}$} & Strongly agree/agree & 91.6 & 86.0 & 81.8 & \multirow[t]{3}{*}{$<0.0001$} \\
\hline & $\begin{array}{l}\text { Neither agree nor } \\
\text { disagree }\end{array}$ & 5.9 & 11.5 & 9.3 & \\
\hline & $\begin{array}{l}\text { Disagree/strongly } \\
\text { disagree }\end{array}$ & 2.5 & 2.5 & 9.0 & \\
\hline \multirow{3}{*}{$\begin{array}{l}\text { I do not have enough time to administer } \\
\text { vaccines to adult patients }\end{array}$} & Strongly agree/agree & 15.8 & 25.1 & 33.5 & \multirow[t]{3}{*}{$<0.0001$} \\
\hline & $\begin{array}{l}\text { Neither agree nor } \\
\text { disagree }\end{array}$ & 31.2 & 19.8 & 35.8 & \\
\hline & $\begin{array}{l}\text { Disagree/strongly } \\
\text { disagree }\end{array}$ & 53.0 & 55.0 & 30.8 & \\
\hline \multirow{3}{*}{$\begin{array}{l}\text { It is difficult to keep track of adult patients' } \\
\text { vaccine status }\end{array}$} & Strongly agree/agree & 70.3 & 68.1 & 83.0 & \multirow[t]{3}{*}{$<0.0001$} \\
\hline & $\begin{array}{l}\text { Neither agree nor } \\
\text { disagree }\end{array}$ & 14.4 & 16.3 & 11.0 & \\
\hline & $\begin{array}{l}\text { Disagree/strongly } \\
\text { disagree }\end{array}$ & 15.3 & 15.6 & 6.0 & \\
\hline \multirow{3}{*}{$\begin{array}{l}\text { I think there should be a national electronic } \\
\text { vaccine registry that keeps track of all the } \\
\text { vaccines that I administer to my patients }\end{array}$} & Strongly agree/agree & 83.7 & 75.4 & 84.8 & \multirow[t]{3}{*}{0.0041} \\
\hline & $\begin{array}{l}\text { Neither agree nor } \\
\text { disagree }\end{array}$ & 11.4 & 18.1 & 10.5 & \\
\hline & $\begin{array}{l}\text { Disagree/strongly } \\
\text { disagree }\end{array}$ & 5.0 & 6.5 & 4.8 & \\
\hline \multirow{3}{*}{$\begin{array}{l}\text { It is important to inform adult patients about } \\
\text { the benefits and risks of adult vaccinations }\end{array}$} & Strongly agree/agree & 96.0 & 91.5 & 93.8 & \multirow[t]{3}{*}{0.2388} \\
\hline & $\begin{array}{l}\text { Neither agree nor } \\
\text { disagree }\end{array}$ & 3.0 & 6.7 & 5.3 & \\
\hline & $\begin{array}{l}\text { Disagree/strongly } \\
\text { disagree }\end{array}$ & 1.0 & 1.8 & 1.0 & \\
\hline \multirow{3}{*}{$\begin{array}{l}\text { It is difficult to keep up with vaccination } \\
\text { recommendations for adults }\end{array}$} & Strongly agree/agree & 54.5 & 54.7 & 64.3 & \multirow[t]{3}{*}{$<0.0001$} \\
\hline & $\begin{array}{l}\text { Neither agree nor } \\
\text { disagree }\end{array}$ & 16.3 & 19.8 & 22.0 & \\
\hline & $\begin{array}{l}\text { Disagree/strongly } \\
\text { disagree }\end{array}$ & 29.2 & 25.5 & 13.8 & \\
\hline \multirow{3}{*}{$\begin{array}{l}\text { It is important to use patient encounters as an } \\
\text { opportunity to ask about their vaccine status }\end{array}$} & Strongly agree/agree & 93.6 & 85.7 & 82.3 & \multirow[t]{3}{*}{0.0065} \\
\hline & $\begin{array}{l}\text { Neither agree nor } \\
\text { disagree }\end{array}$ & 5.4 & 11.9 & 14.5 & \\
\hline & $\begin{array}{l}\text { Disagree/strongly } \\
\text { disagree }\end{array}$ & 1.0 & 2.5 & 3.3 & \\
\hline \multirow{3}{*}{$\begin{array}{l}\text { I do not have adequate storage facilities to } \\
\text { provide adult vaccines }\end{array}$} & Strongly agree/agree & 19.3 & 24.6 & 30.0 & \multirow[t]{3}{*}{0.0002} \\
\hline & $\begin{array}{l}\text { Neither agree nor } \\
\text { disagree }\end{array}$ & 29.7 & 16.8 & 19.8 & \\
\hline & $\begin{array}{l}\text { Disagree/strongly } \\
\text { disagree }\end{array}$ & 51.0 & 58.6 & 50.3 & \\
\hline \multirow{3}{*}{$\begin{array}{l}\text { I am not sufficiently reimbursed to make } \\
\text { offering adult immunisation worthwhile }\end{array}$} & Strongly agree/agree & 12.4 & 43.7 & 52.3 & \multirow[t]{3}{*}{$<0.0001$} \\
\hline & $\begin{array}{l}\text { Neither agree nor } \\
\text { disagree }\end{array}$ & 42.6 & 23.7 & 33.0 & \\
\hline & $\begin{array}{l}\text { Disagree/strongly } \\
\text { disagree }\end{array}$ & 45.0 & 32.6 & 14.8 & \\
\hline
\end{tabular}


Table 2 Continued

\begin{tabular}{|c|c|c|c|c|c|}
\hline Synopsis of statements & Agreement & $\begin{array}{l}\text { Nurses } \\
(\%)\end{array}$ & $\begin{array}{l}\text { Physicians } \\
\text { (\%) }\end{array}$ & $\begin{array}{l}\text { Pharmacists } \\
\text { (\%) }\end{array}$ & p Value \\
\hline \multirow{3}{*}{$\begin{array}{l}\text { I use visits of children as an opportunity to } \\
\text { update vaccinations on accompanying adults }\end{array}$} & Strongly agree/agree & 30.7 & 39.3 & 21.0 & \multirow[t]{3}{*}{$<0.0001$} \\
\hline & $\begin{array}{l}\text { Neither agree nor } \\
\text { disagree }\end{array}$ & 43.6 & 26.4 & 45.8 & \\
\hline & $\begin{array}{l}\text { Disagree/strongly } \\
\text { disagree }\end{array}$ & 25.7 & 34.3 & 33.3 & \\
\hline \multirow{3}{*}{$\begin{array}{l}\text { I have a system in my practice to identify } \\
\text { adults who have not received recommended } \\
\text { vaccines }\end{array}$} & Strongly agree/agree & 34.7 & 34.0 & 11.3 & \multirow[t]{3}{*}{$<0.0001$} \\
\hline & $\begin{array}{l}\text { Neither agree nor } \\
\text { disagree }\end{array}$ & 27.7 & 22.5 & 22.5 & \\
\hline & $\begin{array}{l}\text { Disagree/strongly } \\
\text { disagree }\end{array}$ & 37.6 & 43.5 & 66.3 & \\
\hline \multicolumn{6}{|c|}{ Do you feel the following infections/diseases can have an important impact on the health of adults? } \\
\hline Influenza & Yes & 98.5 & 99.1 & 96.0 & 0.0122 \\
\hline Pertussis & Yes & 84.7 & 86.7 & 82.8 & 0.0762 \\
\hline Pneumonia (pneumococcal) & Yes & 99.5 & 98.8 & 98.0 & 0.4240 \\
\hline Shingles & Yes & 93.6 & 91.3 & 92.8 & 0.8377 \\
\hline \multicolumn{6}{|c|}{ Do you feel the following infections/diseases can be prevented by an adult vaccine? } \\
\hline Influenza & Yes & 76.7 & 94.3 & 87.8 & $<0.0001$ \\
\hline Pertussis & Yes & 79.2 & 92.9 & 88.5 & $<0.0001$ \\
\hline Pneumonia (pneumococcal) & Yes & 80.7 & 91.0 & 91.5 & 0.0003 \\
\hline Shingles & Yes & 59.9 & 85.0 & 83.5 & $<0.0001$ \\
\hline \multicolumn{6}{|c|}{ Do you offer a vaccine to prevent the following infections/diseases? } \\
\hline Influenza & Yes & 86.6 & 98.4 & 73.3 & $<0.0001$ \\
\hline Pertussis & Yes & 48.5 & 70.1 & 26.0 & $<0.0001$ \\
\hline Pneumonia (pneumococcal) & Yes & 67.8 & 95.4 & 66.5 & $<0.0001$ \\
\hline Shingles & Yes & 26.2 & 65.7 & 47.8 & $<0.0001$ \\
\hline \multicolumn{6}{|c|}{ Have you received a vaccine to prevent the following infections/diseases? } \\
\hline Influenza & Yes & 84.2 & 96.5 & 82.3 & $<0.0001$ \\
\hline Pertussis & Yes & 58.9 & 54.5 & 44.3 & $<0.0001$ \\
\hline Pneumonia (pneumococcal) & Yes & 30.2 & 26.7 & 20.8 & 0.0022 \\
\hline Shingles & Yes & 5.4 & 8.5 & 6.3 & 0.0809 \\
\hline
\end{tabular}

with the statement that "there should be a vaccine registry that keeps track of all the vaccines I received so that any doctor in any hospital in the country can have access to my records in order to provide care for me."

\section{Focus groups}

Forty-five HCPs and 62 members of the general public participated in focus groups/interviews across the country. Traditional focus groups were carried out in Prince Edward Island (Charlottetown), British Columbia (Vancouver), Ontario (Toronto and Sudbury), Quebec (Montreal) and Saskatchewan (Regina). Virtual online focus groups included HCP from Ontario, Saskatchewan, Quebec, British Columbia and Alberta, while one-on-one interviews included physicians from Nova Scotia, Ontario and British Columbia. Of the HCPs, $16(36 \%)$ were family physicians, 12 pharmacists $(27 \%), 11(24 \%)$ nurses, $2(4 \%)$ general internists and 2 (4\% paediatricians). One public health physician and one emergency room physician were also interviewed. Focus groups with members of the general public were held in Prince Edward Island (Charlottetown, $n=8$ participants), Quebec (Montreal, $\mathrm{n}=8$ ), Ontario (Toronto and Sudbury, $n=8$ and $n=10$ respectively), Saskatchewan
(Regina, $\mathrm{n}=8$ ) and British Columbia (Vancouver, $\mathrm{n}=7$ ). Two online virtual focus groups were completed in Quebec, Nova Scotia, Prince Edward Island, Alberta and British Columbia ( $n=10 ; 2$ each), Ontario, Quebec and Manitoba $(n=3 ; 1$ each). A total of 32 women and 30 men comprised the focus groups.

Four main themes emerged from the HCP and public focus groups: trust versus mistrust, individual versus society, logistical challenges and vaccine hesitancy.

\section{Trust versus mistrust}

Overall attitudes towards adult immunisation were favourable, with most HCPs and the public acknowledging that prevention is better than treatment. HCPs stated that adult vaccinations are beneficial and necessary in order to prevent the spread of disease, reduce overall morbidity and mortality and protect society and individuals at risk. Most practitioners place trust in those who recommend vaccines such as the Chief Medical Officers of Health, public health practitioners, medical journals and other trusted officials.

Vaccination plays an important role in preventing diseases that can result in serious sickness or even death. $(\mathrm{ON}-\mathrm{HCP})$ 


\begin{tabular}{|c|c|c|c|}
\hline Synopsis of statements & $\begin{array}{l}\text { Strongly } \\
\text { agree/agree } \\
(\%)\end{array}$ & $\begin{array}{l}\text { Neither agree } \\
\text { nor disagree } \\
(\%)\end{array}$ & $\begin{array}{l}\text { Disagree/strongly } \\
\text { disagree (\%) }\end{array}$ \\
\hline It is important for adults to receive all recommended vaccines & 57.5 & 28.0 & 14.5 \\
\hline Vaccines are more important for children than adults & 48.3 & 27.9 & 23.8 \\
\hline Vaccines that are recommended for adults should be publicly funded & 78.1 & 15.6 & 6.4 \\
\hline My doctor/nurse has informed me of the vaccines I should receive & 25.0 & 26.1 & 48.9 \\
\hline I keep a record of what vaccines I have received & 36.6 & 20.2 & 43.2 \\
\hline $\begin{array}{l}\text { According to public health recommendations, I know what vaccines } \\
\text { I am supposed to have received }\end{array}$ & 21.7 & 33.4 & 44.9 \\
\hline \multirow{2}{*}{$\begin{array}{l}\text { I think there should be a vaccine registry that keeps track of all of the } \\
\text { vaccines I received so that any doctor in any hospital in the country } \\
\text { can have access to my records in order to provide care for me. }\end{array}$} & 75.8 & 16.2 & 8.0 \\
\hline & Yes (\%) & No (\%) & I don’t know (\%) \\
\hline \multicolumn{4}{|c|}{ Do you feel the following infections/diseases can have an important impact on the health of adults? } \\
\hline Influenza & 84.2 & 10.5 & 5.3 \\
\hline Pertussis & 56.3 & 8.8 & 34.8 \\
\hline Pneumonia (pneumococcal) & 88.8 & 3.8 & 7.4 \\
\hline Shingles & 74.0 & 9.1 & 16.9 \\
\hline \multicolumn{4}{|c|}{ Do you feel the following infections/diseases can be prevented by an adult vaccine? } \\
\hline Influenza & 60.1 & 25.3 & 14.6 \\
\hline Pertussis & 54.4 & 8.1 & 37.5 \\
\hline Pneumonia (pneumococcal) & 43.0 & 26.4 & 30.6 \\
\hline Shingles & 36.3 & 19.6 & 44.1 \\
\hline \multicolumn{4}{|c|}{ Have you been offered a vaccine to prevent the following infections/diseases? } \\
\hline Influenza & 77.4 & 19.0 & 3.6 \\
\hline Pertussis & 10.8 & 75.6 & 13.6 \\
\hline Pneumonia (pneumococcal) & 17.8 & 72.0 & 10.2 \\
\hline Shingles & 7.9 & 80.0 & 12.1 \\
\hline \multicolumn{4}{|c|}{ Has your healthcare provider recommended that you receive a vaccine to prevent the following infections/diseases? } \\
\hline Influenza & 52.8 & 42.8 & 4.4 \\
\hline Pertussis & 5.6 & 86.1 & 8.3 \\
\hline Pneumonia (pneumococcal) & 13.8 & 78.4 & 7.7 \\
\hline Shingles & 5.1 & 86.4 & 8.6 \\
\hline \multicolumn{4}{|l|}{ Have you received a vaccine to prevent the following infections/diseases? } \\
\hline Influenza & 56.2 & 40.0 & 3.8 \\
\hline Pertussis & 14.1 & 70.3 & 15.6 \\
\hline Pneumonia (pneumococcal) & 15.5 & 72.7 & 11.7 \\
\hline Shingles & 7.5 & 79.8 & 12.7 \\
\hline \multicolumn{4}{|c|}{ If your healthcare provider recommended it, would you receive a vaccine to prevent the following infection/disease? } \\
\hline Influenza & 59.0 & 27.5 & 13.5 \\
\hline Pertussis & 55.0 & 17.6 & 27.4 \\
\hline Pneumonia (pneumococcal) & 59.7 & 17.9 & 22.4 \\
\hline Shingles & 55.0 & 20.2 & 24.8 \\
\hline \multicolumn{4}{|c|}{$\begin{array}{l}\text { Even if you had to pay for the vaccine (around } \$ 100 / \text { dose), would you receive a vaccine to prevent the following infections/ } \\
\text { diseases, if your healthcare provider recommended it? }\end{array}$} \\
\hline Influenza & 15.4 & 63.3 & 21.3 \\
\hline Pertussis & 11.4 & 57.9 & 30.7 \\
\hline Pneumonia (pneumococcal) & 15.7 & 56.5 & 27.8 \\
\hline Shingles & 13.2 & 57.3 & 29.4 \\
\hline
\end{tabular}

Realistically, I don't know if any of us do any primary literature searches at this level. We depend often on the bodies that do make the recommendations and put out the guidelines, like the WHO, or whoever. (PEI-HCP)

Many members of the public also said that adult vaccines are generally helpful and necessary. They considered vaccine side effects as being less dangerous than the disease itself.
... It's probably still better than what could happen to you if you haven't got the shot. (QC-Public)

I fully believe in vaccines for children and adults..... I do believe that the benefits have outweighed the risk. (PEI-Public)

Some HCPs stated that they do not trust vaccine recommendations from pharmaceutical companies and 
some governmental agencies and exhibited overall skepticism about adult vaccination especially in regards to vaccine efficacy and side effects. Those participants suggested that there are too many vaccines and that the public fear multiple injections.

I think there is a lot of vaccine fatigue out there. (ON-HCP)

Some HCPs felt that adult vaccination guidelines are biased opinions by 'trusted experts' not backed by scientific evidence.

My concern.....and I expect that NACI is similar to these others, they are a consensus group...what they are doing is bringing in a bunch of experts together, pooling opinions, and then calling that a guideline. Rather than going to grade A evidence, showing that there is a significant risk or benefit to a particular issue. So I don't think there are really any good randomized trials or strong epidemiological studies. That is my greatest concern with a lot of groups coming out now, making particular recommendations. It's a bunch of experts sitting around a table saying do this and do that (ON-HCP).

Many members of the public also questioned the intentions of the government and pharmaceutical companies related to the development and delivery of vaccines; vaccine mistrust was heightened after the H1N1 campaign.

The government bureaucracy has kind of strained peoples trust when it comes to public health and vaccines. (AB-Public)

My estimation of public health really dropped in the whole H1N1 roll out, and the way that it was communicated. You know there were shortages and just so many things happening with the $\mathrm{H} 1 \mathrm{~N} 1$ vaccine. You sort of have to stand back and say is this going to happen all over again. (ON-Public)

\section{Individual versus society}

There was some polarity in how HCPs and the public think about the philosophy and objectives of immunisation. Many stated that adult vaccination should be an individual decision with emphasis on autonomy, while others emphasised the good of society. Many HCPs stated that disease risk and need for vaccination should be considered by each individual contemplating vaccination.

The choice should be tailored to the individual need. (NS-HCP)

\section{Depends on risk factors. (QC-HCP)}

Similarly, some members of the public believed vaccination is warranted only in certain circumstances and stated that adult vaccination is only helpful for people at high risk. For instance, if participants believed that a vaccine-preventable disease did not pose a direct health threat to them, they refused the vaccine.

Good for some people, but most have a good, immunized system. (PEI-Public).

Good idea I personally haven't had those shots.... for me it's not warranted.... give it to people that need it more than I do.. I'm not very exposed to that and I don't need them... (MB-Public)

For some members of the public, perceptions regarding adult vaccination varied according to vaccine type.

Vaccination is useful as an adult but it depends on the kind of vaccine. (ON-Public)

Depends on what it is for. (PEI-Public)

At the other end of the spectrum, many HCPs and members of the public mentioned that adults should also consider the 'societal good' when making the decision to accept or reject vaccines.

Individuals have a responsibility to the whole society. (ON-HCP)

Yes a personal choice but there is responsibility. (PEI-HCP)

I have a strong opinion, I think everyone should be vaccinated, it is for the social good, and the more people are vaccinated the less probability of transmission. I encourage my kids to do it and my friends. (ON-Public)

I definitely think they are a good idea, selfish side says I want to protect myself and there is also the fear of spreading it to the more susceptible children and seniors and sick so it is definitely a good idea. (NS-Public)

The importance of societal responsibility was emphasised in the context of an aging population.

As the population get older it's important to maintain immunity for the society in general. (NS-HCP)

\section{Logistical challenges}

Two logistical subthemes emerged from the HCP focus groups: the first involving the individual, and the second related to heath systems and vaccine delivery. Many HCPs discuss adult vaccination with their patients but others only do so when they feel vaccination is warranted (people at risk, who travel, who work in specific occupations where disease can be transmitted or where there is a high risk for exposure, and during outbreaks). Travel vaccines seem to be a priority for adults and HCPs stated that many members of the Canadian public simply do not care about adult vaccination in general. Lack of time was described as the main reason why physicians do not discuss vaccination during 
routine visits, as vaccine-related counselling is very time consuming.

I take travel vaccines because there are some diseases I don't want to have. (QC-Public)

If they don't know the vaccine it seems like they don't care. (ON-HCP)

It's a very long discussion and habitually we don't have the time. (BC-HCP)

The biggest problem for a lot of family doctors it's the time, it takes a lot of time, it's not that they don't want to educate the patient, they don't have the time for it. That's a big constraint. (ON-HCP)

Some HCPs utilise annual physical examinations or other issue-related visits as a means to assess the vaccination status of adults.

During the annual physical, nurses identify anyone missing any vaccines. (ON-HCP)

It's part of medical history to check previous immunization as well as previous medication. (NS-HCP)

When people come in with a bite or injuries, I double check that they are up to date. (QC-HCP)

When we see a new mom we ask about Tdap vaccine, with a new baby I recommend the flu vaccine to the parents. (ON-HCP)

Many HCPs believe it is their responsibility to advocate for and educate patients about issues pertaining to adult vaccination. Being in a trusted position allows the HCP to influence patient decision-making which has the potential to 'sway people who are on the fence.' (ON-HCP)

From a logistical perspective, HCPs also understand that many Canadians 'fall through the cracks' after they leave the school system. They state that many members of the public are certain that all vaccines that are pertinent were administered to them as children and that there is no need for additional vaccines or boosters.

When I was a child in school I got all my shots so I'm ok (ON-HCP)

For routine immunization adults assume it has been done. (NS-HCP)

HCPs believe that the general public does not track their vaccination status nor do HCPs have access to an electronic vaccination tracking system. Lack of tracking systems leads to confusion in terms of knowing who is up to date with their vaccines and when the next vaccines are due to be delivered.

You know I think it's a problem though. Because there's no system to keep track of immunizations, it's a piecemeal approach. Often you know, people come in and say I'm going south. So should I need this or that? But there should be a system, a central registry to identify who needs what vaccine. (ON-HCP)

Others simply do not have adequate access to vaccines as they do not have a family physician or visit their doctors on a regular basis.

There are a lot of people who don't go to doctors. There are a lot of people who don't access public healthcare. (NS-HCP)

\section{Vaccine hesitancy}

Perhaps surprisingly, vaccine hesitancy (delay in acceptance or complete refusal of one or more vaccines $)^{17}$ was not a dominant theme in the focus groups, although it was mentioned by a number of participants. Some HCPs state that the public appears to exhibit a moderate degree of vaccine hesitancy which is driven by lack of information or reliance on anecdotal evidence or emotion, and that practitioners are not effectively dispelling vaccination myths.

Some people are still convinced that MMR causes autism... their friends said something that happened with some shot so they are not taking it... or they read something on the internet so they are not taking it. (ON-HCP)

Okay, any kind of medical treatment is a personal choice, but from my standpoint there is a lot of anti-vaccine information, and I don't think that Health Canada or practitioners are doing a great job in terms of countering the myths with correct information. (SK-HCP)

Members of the public who refuse vaccines did not think about protecting those around them and stated that no situation would warrant vaccine acceptance. Those that consistently refuse vaccines did so because they believe that natural immunity is better and that vaccines have the potential to cause disease. Some also mentioned the fear of needles and of adverse events.

I don't generally get them because I find that your natural immunity is best. (BC-Public)

I don't get vaccinated, vaccines give the disease. (QC-Public).

With flu shots I had a really really bad reaction 2 years ago. I do think they are a good thing however but not for myself because of these 2 reactions I had. (BC-Public)

\section{DISCUSSION}

Adult vaccination programmes have not achieved the same degree of success as childhood vaccination programmes, resulting in continued morbidity and mortality from vaccine-preventable diseases. Low vaccine coverage among adults is due to multiple factors, and 
there are many challenges and barriers that must be overcome to improve the situation. ${ }^{9} 18$ This study describes the knowledge, attitudes, beliefs and behaviours of the Canadian public and healthcare providers regarding general issues of adult immunisation and specific information related to four diseases and the associated vaccines recommended for routine use in adults (influenza, pertussis, pneumococcus, zoster). Similar to recent findings in the USA, we found that the barriers and facilitators to adult immunisation are interrelated and one barrier may be the direct cause of another. For example, lack of trust or knowledge of a vaccine may be the result of the physician lacking time to educate and recommend adult vaccines to their patients. The US National Vaccine Advisory Committee (NVAC) also suggested that barriers to immunisation do not fall into neatly organised categories and can vary across the lifespan and according to the health condition and the life situation of adults. ${ }^{10} 19$

We found that a substantial proportion of the Canadian public believes that vaccines are more important for children, while only slightly more than 50\% believe it is important to receive all recommended adult vaccines. Lack of knowledge about personal adult vaccination status was also identified. Many focus group participants believed that all relevant vaccines were administered in childhood and there was low awareness of the need for adult vaccines. Lack of awareness of vaccine status among adults has been previously identified as an issue in achieving high vaccine coverage. ${ }^{20} 21$ While HCPs displayed a greater awareness of infectious disease burden and the need for adult vaccines, we found that many of the attitudes and beliefs identified were disease- and vaccine-specific. For instance, $92 \%$ of HCPs believed that influenza, pneumococcal pneumonia and shingles could have an important impact on the health of adults while only $82.8-86.7 \%$ believed that about pertussis. In contrast, fewer members of the public believed these diseases had an important impact on health (influenza, $84.2 \%$; shingles, $74 \%$; pneumonia, $88.8 \%$; pertussis $56.3 \%$ ). Similar to other published data, ${ }^{19} 22$ the focus groups suggested that decisions about acceptance of vaccination were dependent on vaccine type, disease risk and current health status.

Trust or mistrust of vaccines was identified in our survey and focus groups. Trust in vaccines was higher among HCPs than the public, but mistrust concerning the intentions of pharmaceutical companies as well as the government was identified by both groups. Skepticism regarding vaccine adverse events and efficacy as well as the development and delivery of vaccines were cited as the main reasons for this mistrust. Some participants commented that their level of mistrust increased after the H1N1 epidemic. These findings are supported by previous studies demonstrating that adults are apprehensive about the possibility of adverse events and are concerned that vaccines can actually cause clinical infection. $^{20-26}$ We identified divergent views among the
Canadian public related to the philosophical rationale for the use of vaccines. Some participants believed the decision only to be personal and focused on autonomy, while others emphasised vaccination for the good of society. The notion of increased social responsibility was emphasised in the context of the aging population. While there is little in the literature regarding this dichotomy in relation to the general public, much has been written in this regard related to vaccination of HCPs with influenza vaccine to prevent transmission of influenza to high-risk patients. ${ }^{27-29}$ Even with high levels of knowledge and awareness about the risk of transmission of infection to patients, there is great variability among HCPs as to whether their primary motivation for immunisation is to protect themselves or their patients. ${ }^{30-33}$

In addition to issues related to knowledge, attitudes and beliefs, logistical challenges were cited as reasons for low adult immunisation rates. In the survey, we found that adult vaccines are not being routinely recommended by HCPs, while members of the public indicates willingness to get immunised if their HCP recommends it. Participants in our focus groups indicated that HCPs discuss adult immunisation with their patients, but may only do so when they feel vaccination is warranted such as for travel, which was identified as a high priority, and for people who are at risk. Those HCPs who do not discuss adult vaccination at routine visits cite time constraints as the main driver for this behaviour. At a health system level, the lack of electronic tracking systems was identified as the greatest barrier, although lack of physician access was cited when electronic systems are in place. This has also been identified as barrier to optimal paediatric vaccine coverage in Canada. ${ }^{34}$

In the USA, lack of coordination of adult immunisation activities due to inadequate infrastructure has been identified as a major contributor to the lack of promotion of adult vaccines. ${ }^{10}$ For instance, some adults may have difficulty accessing a consistent primary care provider and often seek care at a variety of locations such as travel and after-hour clinics or pharmacies. Receiving vaccines at a variety of locations without the proper communication between facilities can lead to gaps in preventative services. In addition, time constraints often prevent physicians from following through with preventative service recommendations such as vaccine counselling. ${ }^{35} 36$ The large number of preventative recommendations and the high patient volume contribute to this burden. ${ }^{36}$

Time spent on preventative service and vaccine counselling is critical as members of the public are strongly influenced by the recommendation of a HCP to receive vaccines. ${ }^{3}$ The National Immunization Coverage Survey found that for both seasonal influenza and pneumococcal vaccines a recommendation by a HCP was the strongest predictor of vaccine acceptance; ${ }^{3}$ in the USA, $87 \%$ of survey respondents were likely or somewhat likely to get adult vaccines if recommended by their doctor. ${ }^{10}$ 
Other studies have found similar results. ${ }^{20} \quad \begin{array}{llll}23 & 37 & 38\end{array}$ Postpartum women were willing to receive vaccination if recommended by a HCP but only $5-10 \%$ actually reported having these discussions with their providers. ${ }^{39}$ Physicians who do not recommend or provide influenza or pneumococcal vaccines indicate that they lack engagement in preventative care as they focus on urgent healthcare issues during office visits. ${ }^{40}$ Other barriers include cost of purchasing vaccines, the low level of remuneration for vaccination, the difficulty of reaching nonvaccinated patients and the availability of physicians and nurses. ${ }^{41}$ In contrast, physician perceptions as to why adults don't receive tetanus, influenza or pneumococcal vaccines include patients' failure to schedule wellpatient visits, lack of effective reminder systems, people not going to their family doctor and lack of time during doctor visits. ${ }^{20}$ Other than time constraints, providers may not also recommend vaccines if they do not stock the vaccines in their office. ${ }^{42}$

Financial issues were not identified in our study as a major concern. Similar proportions of participants agreed that recommended adult vaccines should be publicly funded $(81.8-91.6 \%$ of HCPs and $78.1 \%$ of the public). Less than $16 \%$ of the Canadian public would be willing to pay $\$ 100$ for any of the four adult vaccines discussed. In other studies, financial barriers are not frequently cited as a barrier to receiving adult vaccines. $^{20} 22$ Cost, however, was identified as a barrier in other studies, ${ }^{43} 44$ and the likelihood of being vaccinated was greater if they were available at little to no cost. ${ }^{45}$

In the USA, a roadmap for improving vaccine coverage among adults has been developed, with the plan to reassess the status of adult vaccine coverage once the interventions have been put into place. ${ }^{10}$ The NVAC recommendations include national leadership to establish an adult immunisation programme to parallel the existing US childhood immunisation programme, provision of appropriate financial and infrastructure resources to carry out the strategic plan of the newly created adult immunisation programme and establishment of a national strategic plan for adult immunisation. Focused activities to carry out this strategy and plan included setting immunisation goals, establishing infrastructure, expanding access to vaccination, provider education and ongoing monitoring including follow-up assessment of the knowledge, attitudes, beliefs and behaviours of providers and the public in response to these interventions.

Strong leadership and coordination of adult immunisation programmes are required in order to address the challenge of low adult immunisation rates. ${ }^{46}$ This coordination is essential as adults access many providers and lack information regarding the existence and importance of these vaccines. In addition, adult vaccines are targeted to a more diverse age range, making delivery and promotion of vaccines more problematic. The infrastructure used to deliver adult vaccines differs substantially from that of the paediatric population and this must be acknowledged in order to move towards a comprehensive life-long vaccination programme that is even more critical as our population ages. In Canada, no such coordinated effort has been initiated to evaluate and improve immunisation among adults. This study begins to address some of the recommendations suggested by the US NVAC, identifying some of the gaps in the literature especially as it pertains to the key barriers that must be overcome to increase adult vaccination rates, and can provide an important component of a baseline assessment in advance of establishing a Canadian national strategy for adult immunisation.

\section{Author affiliations}

${ }^{1}$ Canadian Center for Vaccinology, Dalhousie University, IWK Health Centre and Nova Scotia Health Authority, Nova Scotia, Canada

${ }^{2}$ School of Nursing, St. Francis Xavier University, Nova Scotia, Canada

${ }^{3}$ School of Nursing, Dalhousie University, Nova Scotia, Canada

${ }^{4}$ Department of Pediatrics, Dalhousie University, Nova Scotia, Canada

${ }^{5}$ Department of Medicine, Dalhousie University, Nova Scotia, Canada

${ }^{6}$ Department of Community Health and Epidemiology, Dalhousie University,

Nova Scotia, Canada

${ }^{7}$ Department of Microbiology \& Immunology, Dalhousie University, Nova Scotia, Canada

Acknowledgements The authors thank Bruce Smith for his assistance with the statistical analysis and Kristine Webber for her assistance with the thematic analysis of the focus groups.

Contributors DMM and SAH wrote the first draft of the manuscript and other authors reviewed subsequent drafts and provided comments. DMC and LL were responsible for the statistical analysis. All authors contributed to the design of the study and the analysis of the data. All authors approved the final submitted versions.

Funding This study was funded by research grants from GlaxoSmithKline and Sanofi Pasteur. The funders played no role in the collection or analysis of the data.

Competing interests SAH, SAM and JML have received grants and contracts for clinical trials and epidemiological studies from GlaxoSmithKline and Sanofi Pasteur and have served on ad hoc scientific advisory panels for the two manufacturers.

Ethics approval IWK Health Centre, Halifax, NS, Canada.

Provenance and peer review Not commissioned; externally peer reviewed.

Data sharing statement The authors will consider requests for sharing of the raw data from this study on a case-by-case basis.

Open Access This is an Open Access article distributed in accordance with the Creative Commons Attribution Non Commercial (CC BY-NC 4.0) license, which permits others to distribute, remix, adapt, build upon this work noncommercially, and license their derivative works on different terms, provided the original work is properly cited and the use is non-commercial. See: http:// creativecommons.org/licenses/by-nc/4.0/

\section{REFERENCES}

1. Poland GA, Belmin J, Langley J, et al. A global prescription for adult immunization: time is catching up with us. Vaccine 2010;28:7137-9.

2. Public Health Agency of Canada. Vaccine coverage in Canadian children: Results from the 2011 Childhood National Immunization Coverage Survey. http://www.phac-aspc.gc.ca/im/nics-enva/ vccc-cvec-eng.php (accessed 29 May 2015).

3. Public Health Agency of Canada. Vaccine coverage amongst adult Canadians: Results from the 2012 adult National Immunization Coverage (aNIC) survey. http://www.phac-aspc.gc.ca/im/nics-enva/ vcac-cvac-eng.php (accessed 20 May 2015). 
4. Williams WW, Lu PJ, O'Halloran A, et al. Vaccination coverage among adults, excluding influenza vaccination-United States, 2013. MMWR Morb Mortal Wkly Rep 2015;64:95-102.

5. National Center for Immunization and Respiratory Diseases. General recommendations on immunization-recommendations of the Advisory Committee on Immunization Practices (ACIP). MMWR Recomm Rep 2011;60(RR-2):1-64.

6. Public Health Agency of Canada. Canadian Immunization Guide. Ottawa, ON: Public Health Agency of Canada, 2012 (updated 24 Apr 2014; cited 9 Mar 2015). http://www.phac-aspc.gc.ca/publicat/ cig-gci/p01-12-eng.php

7. Public Health Agency of Canada. Publicly-funded immunization programs in the provinces and territories of Canada: Routine and high risk schedule for adults (as of September 2014). http://www. phac-aspc.gc.ca/im/ptimprog-progimpt/table-3-eng.php; (accessed 29 May 2015)

8. Wheelock A, Thomson A, Sevdalis N. Social and psychological factors underlying adult vaccination behavior: lessons from seasonal influenza vaccination in the US and the UK. Expert Rev Vaccines 2013;12:893-901.

9. Swanson $\mathrm{KA}$, Schmitt $\mathrm{HJ}$, Jansen $\mathrm{KU}$, et al. Adult vaccination. Hum Vaccin Immunother 2015;11:150-5.

10. National Vaccine Advisory Committee. A pathway to leadership for adult immunization: recommendations of the National Vaccine Advisory Committee: approved by the National Vaccine Advisory Committee on June 14, 2011. Public Health Rep 2012;127(Suppl 1):1-42.

11. Ivankova NV, Stick SL. Students' persistence in a distributed doctoral program in educational leadership in higher education: a mixed methods study. Res Higher Educ 2007;48:93-135.

12. Creswell JW. Education research: planning, conducting, and evaluating quantitative and qualitative approaches to research. 2nd edn. Upper Saddle River, NJ: Merrill/Pearson Education, 2005.

13. MacDougall D, Halperin BA, MacKinnon-Cameron D, et al. Universal tetanus, diphtheria, acellular pertussis (Tdap) vaccination of adults: what Canadian health care providers know and need to know. Hum Vaccin Immunother 2015;11:2167-79.

14. Tong A, Sainsbury P, Craig J. Consolidated criteria for reporting qualitative research (COREQ): a 32 -item checklist for interviews and focus groups. Int J Qual Health Care 2007;19:349-57.

15. Pathman DE, Konrad TR, Freed GL, et al. The awareness to adherence model of the steps to clinical guideline compliance. The case of pediatric vaccine recommendations. Med Care 1996;34:873-89.

16. Krueger RA, Casey MA. Focus groups: a practical guide for applied research. 4th edn. Thousand Oaks, CA: Sage Publications, Inc., 2009.

17. Dubé E, Gagnon D, MacDonald NE, SAGE Working Group on Vaccine Hesitancy. Strategies intended to address vaccine hesitancy: review of published reviews. Vaccine 2015;33:4191-203.

18. Baeyens JP. Ensuring the willingness to vaccinate and be vaccinated. Expert Rev Vaccines 2010;9(3 Suppl):11-14.

19. Wheelock A, Parand A, Rigole B, et al. Socio-psychological factors driving adult vaccination: a qualitative study. PLOS ONE 2014;9: e113503.

20. Johnson DR, Nichol KL, Lipczynski K. Barriers to adult immunization. Am J Med 2008;121(7 Suppl 2):S28-35.

21. National Foundation for Infectious Diseases. National survey on adult vaccination reports low consumer awareness of vaccines and the risks of vaccine preventable diseases. Bethesda, MD: National Foundation for Infectious Diseases, 2008.

22. Madjid M, Alfred A, Sahai A, et al. Factors contributing to suboptimal vaccination against influenza: results of a nationwide telephone survey of persons with cardiovascular disease. Tex Heart Inst $J$ 2009;36:546-52.

23. Lindley MC, Wortley PM, Winston CA, et al. The role of attitudes in understanding disparities in adult influenza vaccination. Am J Prev Med 2006;31:281-5
24. Mayo AM, Cobler S. Flu vaccines and patient decision making: what we need to know. J Am Acad Nurse Pract 2004;16:402-10.

25. Schneider EC, Cleary PD, Zaslavsky AM, et al. Racial disparity in influenza vaccination: does managed care narrow the gap between African Americans and whites? JAMA 2001;286:1455-60.

26. Bardenheier BH, Wortley PM, Winston CA, et al. Do patterns of knowledge and attitudes exist among unvaccinated seniors? Am J Health Behav 2006;30:675-83.

27. Begue RE, Gee SQ. Improving influenza immunization among healthcare workers. Infect Control Hosp Epidemiol 1998;19:518-20.

28. Manuel DG, Henry B, Hockin J, et al. Health behavior associated with influenza vaccination among healthcare workers in long-term-care facilities. Infect Control Hosp Epidemiol 2002;23:609-14.

29. Nichol KL, Hauge M. Influenza vaccination of healthcare workers. Infect Control Hosp Epidemiol 1997;18:189-94.

30. Heimberger T, Chang HG, Shaikh M, et al. Knowledge and attitudes of healthcare workers about influenza: why are they not getting vaccinated? Infect Control Hosp Epidemiol 1995;16:412-15.

31. Ballada D, Biasio LR, Cascio G, et al. Attitudes and behavior of health care personnel regarding influenza vaccination. Eur $J$ Epidemiol 1994;10:63-8.

32. Hammer G. Factors associated with hepatitis B vaccine acceptance among nursing home workers. [Doctoral dissertation]. Johns Hopkins University, 1997. Dissertation Abstracts International (UMI NO 9821130)

33. Everett WW, Coffin SE, Zaoutis T, et al. Smallpox vaccination: a national survey of emergency health care providers. Acad Emerg Med 2003;10:606-11.

34. Eggertson L. Experts call for national immunization registry, coordinated schedules. CMAJ 2011;183:E143-4.

35. Yarnall KS, Pollak KI, Østbye T, et al. Primary care: is there enough time for prevention? Am J Public Health 2003;93:635-41.

36. Pollak KI, Krause KM, Yarnall KS, et al. Estimated time spent on preventive services by primary care physicians. BMC Health Serv Res 2008;8:245.

37. Suryadevara M, Bonville CA, Cibula DA, et al. Pertussis vaccine for adults: knowledge, attitudes, and vaccine receipt among adults with children in the household. Vaccine 2014;32:7000-4.

38. Wiley KE, Massey PD, Cooper SC, et al. Pregnant women's intention to take up a post-partum pertussis vaccine, and their willingness to take up the vaccine while pregnant: a cross sectional survey. Vaccine 2013;31:3972-8.

39. Beel ER, Rench MA, Montesinos DP, et al. Knowledge and attitudes of postpartum women toward immunization during pregnancy and the peripartum period. Hum Vaccin Immunother 2013;9:1926-31.

40. Szilagyi PG, Shone LP, Barth R, et al. Physician practices and attitudes regarding adult immunizations. Prev Med 2005;40:152-61.

41. Huot C, Sauvageau C, Tremblay G, et al. Adult immunization services: steps have to be done. Vaccine 2010;28:1177-80.

42. Freed GL, Clark SJ, Cowan AE, et al. Primary care physician perspectives on providing adult vaccines. Vaccine 2011;29:1850-4.

43. Fowles JB, Beebe TJ. Failure to immunize the elderly: a systems problem or a statement of personal values? Jt Comm J Qual Improv 1998;24:704-10.

44. Mortensen GL. Perceptions of herpes zoster and attitudes towards zoster vaccination among 50-65-year-old Danes. Dan Med Bull 2011;58:A4345.

45. Cameron KA, Rintamaki LS, Kamanda-Kosseh M, et al. Using theoretical constructs to identify key issues for targeted message design: African American seniors' perceptions about influenza and influenza vaccination. Health Commun 2009;24:316-26.

46. National Foundation for Infectious Diseases. Call to action: adult vaccination saves lives. Bethesda, MD: National Foundation for Infectious Diseases, 2012. 